\title{
NONCONGRUENT EQUIDISSECTIONS OF THE PLANE
}

\author{
D. FRETTLÖH
}

\begin{abstract}
Nandakumar asked whether there is a tiling of the plane by pairwise non-congruent triangles of equal area and equal perimeter. Here a weaker result is obtained: there is a tiling of the plane by pairwise non-congruent triangles of equal area such that their perimeter is bounded by some common constant. Several variants of the problem are stated, some of them are answered.
\end{abstract}

Dedicated to Karoly Bezdek and to my academic brother Egon Schulte on the occasion of their 60th birthday

\section{INTRODUCTION}

There are several problems in Discrete Geometry, old and new, that can be stated easily but are hard to solve. Tilings and dissections provide a large number of such problems, see for instance CFG, Chapter C]. On his blog [N], R. Nandakumar asked in 2014:

Question 1. "Can the plane be filled by triangles of same area and perimeter with no two triangles congruent to each other?"

His webpage $[\mathrm{N}]$ contains several further interesting problems of this flavour. The main result of this paper, Theorem 2 , answers a weaker form of the question above affirmatively. Section 4 is dedicated to the statement and the proof of this result, together with its analogues for quadrangles, pentagons and hexagons. Section 3 formulates several variants of this problem and gives a systematic overview. Section 2 contains some basic observations and a first result on a similar result for quadrangles.

1.1. Notation. A tiling of $\mathbb{R}^{2}$ is a collection $\left\{T_{1}, T_{2}, \ldots\right\}$ of compact sets $T_{i}$ (the tiles) that is a packing of $\mathbb{R}^{2}$ (i.e., the interiors of distinct tiles are disjoint) as well as a covering of $\mathbb{R}^{2}$ (i.e. the union of the tiles equals $\mathbb{R}^{2}$ ). In general, tile shapes may be pretty complicated, but for the purpose of this paper tiles are always simple convex polygons. A tiling is called vertex-to-vertex, if the intersection of any two tiles is either an entire edge of both tiles, or a point, or empty. A tiling $\mathcal{T}$ is locally finite if any compact set in $\mathbb{R}^{2}$ intersects only finitely many tiles of $\mathcal{T}$. A tiling $\mathcal{T}$ is normal if there are $R>r>0$ such that (1) each tile in $\mathcal{T}$ contains some ball of radius $r$, and (2) each tile is contained in some ball of radius $R$. By [GS, 3.2.1] we have that each normal tiling is locally finite.

\section{BASIC OBSERVATIONS}

In Question 1 above, "filled" is to be understood in the sense that the plane is covered without overlaps. In other words: is there a tiling of the plane by pairwise noncongruent triangles all having the same area and the same perimeter? If one tries to find a solution one realises that the problem seems to be highly overdetermined. One possibility to relax the problem is to drop the requirement on the perimeter. So one may ask "Is there a tiling of the plane by pairwise noncongruent triangles all having the same area?" It is not too hard to find examples. One possibility is to partition the plane into half-strips and divide these half-strips into triangles, as shown in Figure 1 .

The image indicates how to fill the right half-plane by half-strips made of triangles. If all halfstrips have different widths then all triangles are distinct. The left half-plane can be filled in an

Date: September 23, 2021. 


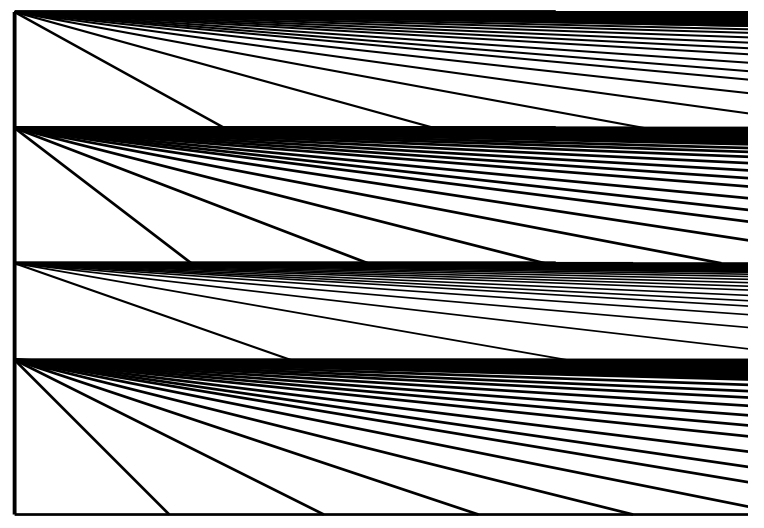

FiguRE 1. Tiling of the plane by pairwise non-congruent triangles of unit area. The perimeters of the triangles are unbounded. Moreover, the tiling is not locally finite.

analogous manner. Anyway, this tiling is not locally finite: the upper vertex of any half-strip is contained in infinitely many triangles. So one may ask "Is there a locally finite tiling of the plane by pairwise noncongruent triangles of unit area?" Even in this stronger form the question was already answered by R. Nandakumar. The image in Figure 2 shows a solution, see also $[\mathrm{W}$. The idea is to dissect the upper right quadrant into triangles of area $\frac{1}{2}$ by zigzagging between the horizontal axis and the vertical axis. The triangles become very long and thin soon. Nevertheless they are filling the quadrant. For the remaining three quadrants one uses an analogous construction, perturbing the coordinates slightly. (For instance, stretch a copy of the first quadrant by some irrational factor $q>1$ in the horizontal direction and shrink it by $\frac{1}{q}$ in the vertical direction. See [W] for an alternative, more detailed explanation.) This tiling is locally finite. Nevertheless, this example is not really satisfying. More precisely, this tiling is not normal, since in this solution the perimeters of the triangles become arbitrary large. (Hence the inradii become arbitrary small). So it seems natural to ask:

Question 2. "Is there a normal tiling of the plane by pairwise noncongruent triangles of unit area?"

This question was already asked by Nandakumar, in the form whether there is a tiling of the plane by pairwise noncongruent triangles all having unit area such that the perimeter of

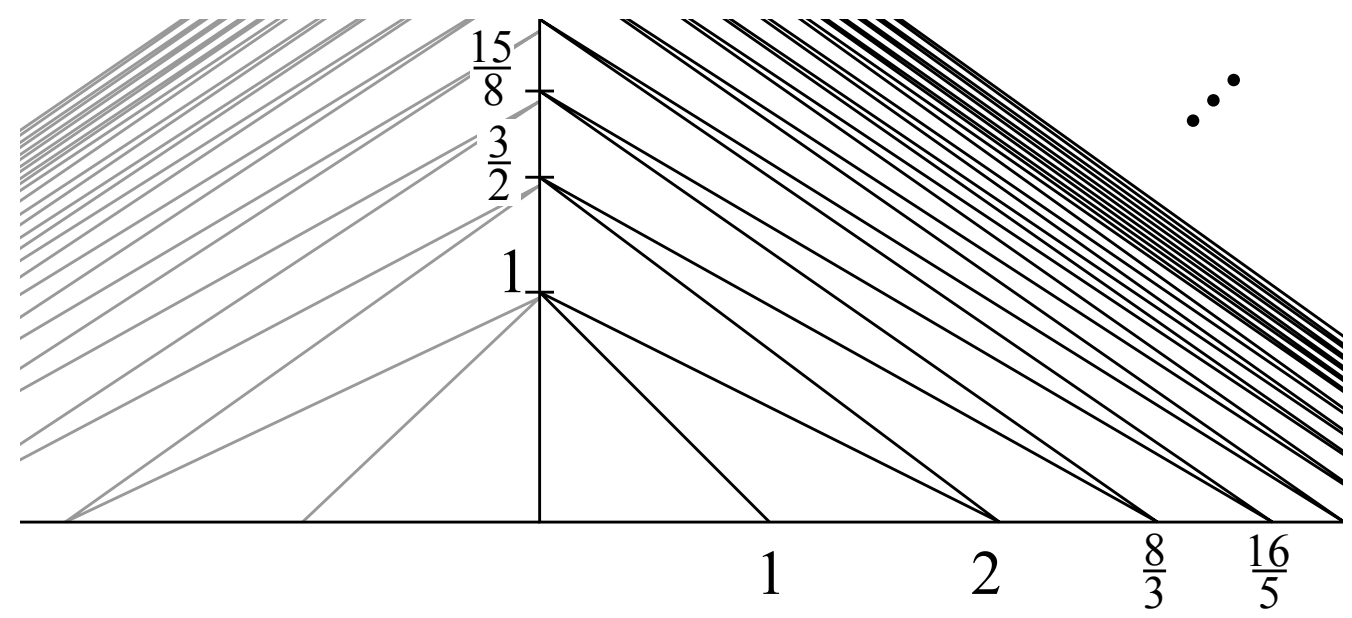

FiguRE 2. Tiling of the plane by pairwise non-congruent triangles of area $\frac{1}{2}$. 


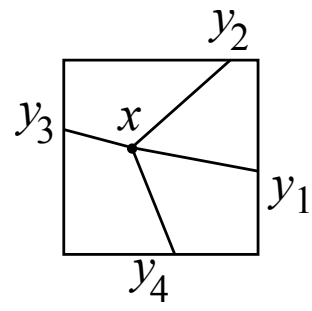

Figure 3. Partitioning a square into 4 distinct quadrangles of equal area. There are uncountably many possibilities for such a dissection.

the triangles is bounded by some common constant. Theorem 2 below answers this question affirmatively.

One possible approach to find a solution is the following. If one can partition a set $S \subset \mathbb{R}^{2}$ into triangles of unit area such that (1) $S$ tiles the plane, and (2) the triangles can be distorted continuously, in a way such that all triangles in $S$ are distinct (but still having unit area), this solves the problem. We will illustrate this concept (where "triangle" is replaced by "quadrangle") in the proof of the following result.

Theorem 1. There is a normal tiling of the plane by pairwise non-congruent convex quadrangles of unit area.

Proof. Consider a square $Q$ of edge length 2. Let $x \in Q$ be a point such that (1) for the distance $d$ of $x$ to the centre of $Q$ holds $0<d<\frac{1}{10}$, and (2) $x$ is neither contained in the diagonals of $Q$ nor in the line segments connecting mid-points of opposite edges of $Q$. Let $y_{1}$ be a point on the boundary of $Q$ such that for the distance $d^{\prime}$ of $y$ to the mid-point of the edge containing $y_{1}$ holds $0<d^{\prime}<\frac{1}{10}$. (Here the requirements on $x$ and $y_{1}$ being close to the centre of the squre resp. the centre of the edge are needed to exclude the possibility of non-convex quadrangles resp. triangles occurring.)

The choice of $x$ and $y_{1}$ determines three further unique points $y_{2}, y_{3}, y_{4}$ on the boundary of $Q$ such that the line segments $x y_{i}(1 \leq i \leq 4)$ partition $Q$ into four quadrangles of unit area. By the choice of $x$, avoiding the mirror axes of $Q$, it can always be achieved that all quadrangles in a single partition of $Q$ are distinct. (In fact the author believes that no two congruent quadrangles can occur in a partition where $x$ is not contained in the mirror axes of $Q$, but this might be tedious to prove. Here we prefer rather to use the free parameters to achieve that all quadrangles are distinct.) Figure 3 indicates such a partition.

The two coordinates determining $x$ can be changed continuously within a small range independently, yielding two free parameters. One coordinate of $y_{1}$ can be changed continuously, too, within some small range. Hence we obtain the desired tiling as follows: Tile the plane $\mathbb{R}^{2}$ with copies of the square $Q$. Dissect each copy of $Q$ into four quadrangles of area 1 , in some order. In each dissection, choose $x$ and $y_{1}$ such that the resulting quadrangles have not shown up earlier in the construction. This is always possible since, in each step, there are only finitely many quadrangles constructed already, whereas there are uncountably many choices for $x$ and $y_{1}$.

At this point it becomes obvious that Questions 1 and 2 lead to several variants. The example in the proof above yields a normal tiling, but in general not one that is vertex-to-vertex. One may ask the questions for triangles, for quadrangles, for pentagons, and in each case with or without requiring "equal perimeter", or "normal", or "vertex-to-vertex". The next section aims to give a systematic overview of the questions.

\section{VARIANTS OF THE PROBLEM}

The general property we will require throughout the paper is that a tiling consists of convex tiles of unit area such that all tiles are pairwise distinct. The tiles can be triangles (as in the original question), but also quadrangles, rectangles, pentagons or hexagons. We may or may not require additionally that all tiles have equal perimeter, or that the perimeter is bounded by some 
common constant, or that the tilings are normal, or just locally finite. Furthermore, it may be possible to construct a tiling analogous to the proof of Theorem 1 that is, by tiling a tile $S$ in infinitely many ways, where $S$ in turn can tile the plane. The connections between these properties is shown in the following diagram.

$$
\left.\begin{array}{ll}
\text { equal perimeter } & \Rightarrow \\
\text { tiling a tile } & \Rightarrow
\end{array}\right\} \text { perimeter is bounded } \Leftrightarrow \text { normal } \Rightarrow \text { locally finite }
$$

For instance, Equation (1) tells that if there is tiling obtained by tiling a tile $S$ in infinitely many ways, then the perimeters of the tiles in this tiling are bounded by some common constant. In turn, the latter is equivalent to the tiling being normal (since all tiles are convex and have unit area), which in turn implies (by GS, 3.2.1]) that the tiling is locally finite.

These implications help to give an overview of the several variants of the questions. The following tables list, for each of the cases of triangles, convex quadrangles, convex pentagons, and convex hexagons, whether there is some tiling known fulfilling the properties in Equation (1), and whether there is such a tiling that is even vertex-to-vertex. Because of the implications in Equation (1), if there is "yes" in some column, then the entries above in the same column contain also a "yes".

Note that "not vtv" is usually a weaker condition than "vtv", but a tiling by convex hexagons that is not vtv is much harder to find than one that is vtv.

\begin{tabular}{l|cc}
\hline Triangles & vtv & not vtv \\
\hline locally finite & $?$ & yes \\
bounded perimeter & $?$ & yes \\
tiling a tile & $?$ & $?$ \\
equal perimeter & $?$ & $?$ \\
\hline Pentagons & vtv & not vtv \\
\hline locally finite & $?$ & yes \\
bounded perimeter & $?$ & yes \\
tiling a tile & $?$ & yes \\
equal perimeter & $?$ & $?$ \\
\hline
\end{tabular}

\begin{tabular}{l|cc}
\hline Quadrangles & vtv & not vtv \\
\hline locally finite & yes & yes \\
bounded perimeter & yes & yes \\
tiling a tile & yes & yes \\
equal perimeter & $?$ & $?$ \\
\hline Hexagons & vtv & not vtv \\
\hline locally finite & yes & $?$ \\
bounded perimeter & yes & $?$ \\
tiling a tile & yes & $?$ \\
equal perimeter & $?$ & $?$ \\
\hline
\end{tabular}

Theorem 1 above proves the case "quadrangles: tiling a tile, not vtv" (thus also the two cases above it in the same column in the corresponding table). Theorem 2 below proves "triangles: bounded perimeter, not vtv", Theorem 3 proves "quadrangles: tiling a tile, vtv", Theorem 4 proves "hexagons: tiling a tile, vtv", and Theorem 5 proves "hexagons: tiling a tile, vtv".

\section{MAin Results}

Theorem 2. There is a normal tiling of the plane by pairwise non-congruent triangles of unit area.

Proof. The idea of the proof is a refinement of the construction in Figure 2. Basically we add additional fault lines in each quadrant. Moreover, we make use of some free parameter in some range, allowing for uncountably many choices, where in each step of the construction only finitely many triangle shapes must be avoided.

Choose some constant $c$ big enough. This serves as the upper bound on the perimeter of the triangles. For our purposes $c=100$ will do. Consider the upper right quadrant $Q_{1}$. Pick a point $x_{0}$ on the positive horizontal axis with $\left|x_{0}\right|<\frac{c}{3}$. Let $T_{1}$ be the unique triangle in $Q_{1}$ with vertices $0, x_{0}$ and area 1. (For this and what follows compare Figure 4. ) Denote the third vertex of $T_{1}$ by $y_{1}$. Choose $y_{2}$ on the horizontal axis such that the triangle $T_{2}$ with vertices $x_{0}, y_{1}$ and $y_{2}$ has area 1. Continue zigzagging in this way between horizontal and vertical axis. I.e., choose $y_{i+1}$ on the axis not containing $y_{i}$ such that the triangle $T_{i+1}$ with vertices $y_{i-1}, y_{i}, y_{i+1}$ has area 1 . Repeat this until the next triangle $T_{i+2}$ would have perimeter larger than $c$. Omit $T_{i+2}$. Pick $x_{1}$ such that the triangle $y_{i}, y_{i+1}, x_{1}$ has area 1 .

There are uncountably many choices for $x_{1}$. For the sake of symmetry let $x_{1}$ be close to the bisector $\{(x, x) \mid x \in \mathbb{R}\}$ of $Q_{1}$. Choose a half-line $\ell_{1}$ proceeding from $x_{1}$. There are uncountably 


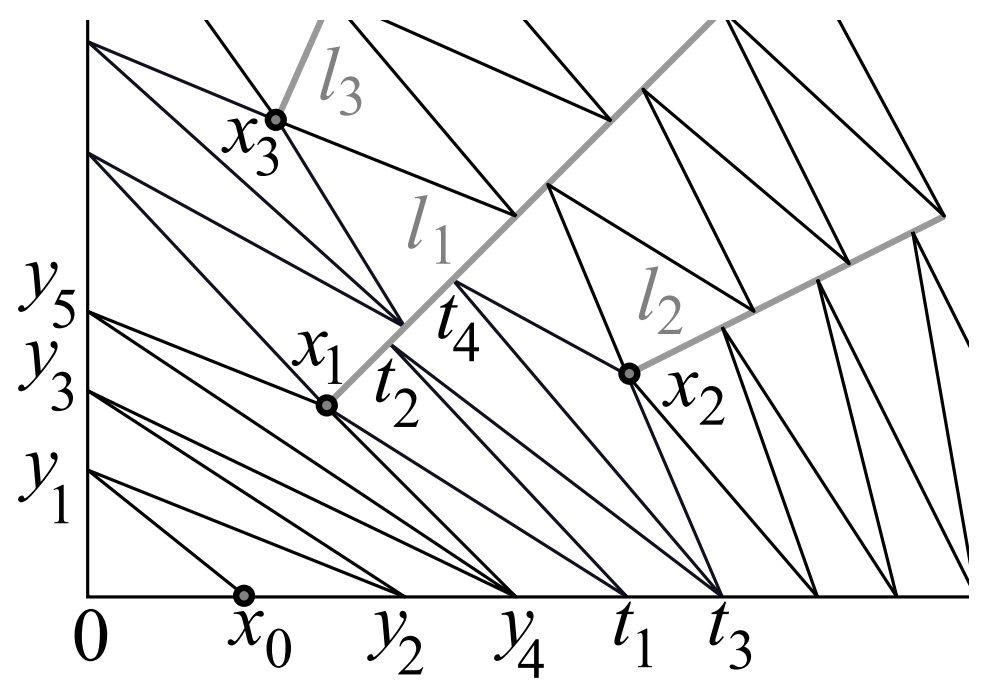

FiguRE 4 . Tiling the upper right quadrant $Q_{1}$ by pairwise non-congruent triangles of unit area and bounded perimeter.

many choices for $\ell_{1}$. Again, for the sake of symmetry, let $\ell_{1}$ be close to the bisector of $Q_{1}$. Continue by zigzagging in two regions, between the horizontal axis and $\ell_{1}$, and between the vertical axis and $\ell_{1}$. I.e., if $y_{2 k}$ is the last point on the horizontal axis, pick $t_{1}$ on the horizontal axis such that the triangle $y_{2 k}, x_{1}, t_{1}$ has area 1 . Continue by choosing $t_{2}$ on $\ell_{1}$ such that the triangle $x_{1}, t_{1}, t_{2}$ has area 1 and so on, until the perimeter of the next triangle $t_{i}, t_{i+1}, t_{i+2}$ would be larger than $c$. Omit this triangle. Choose $x_{2}$ such that the new triangle $t_{i}, t_{i+1}, x_{2}$ has area 1 . Choose a half-line $\ell_{2}$ proceeding from $x_{2}$. Again there are uncountably many choices for $x_{2}$ and $\ell_{2}$.

Do the analogous construction in the upper region between $\ell_{1}$ and the vertical axis. Continue in this manner. Whenever a triangle occurs with perimeter larger than $c$ choose a new point $x_{k}$ and a new line $\ell_{k}$ dividing the old region into two.

The uncountability of choices for $x_{k}$ and $\ell_{k}$ ensures that we can always avoid to add a triangle that is congruent to some triangle added earlier. Indeed, whenever we are in the situation to choose $x_{k}$ and $\ell_{k}$ there are at most countably many triangles constructed already. Hence $x_{k}$ can be chosen such that no triangle with vertex $x_{k}$ is congruent to an already constructed one, and $\ell_{k}$ can be chosen such that no triangle occurring in the two new regions defined by $\ell_{k}$ is congruent to an already constructed one. Hence the quadrant $Q_{1}$ can be tiled by pairwise non-congruent triangles with area 1 and perimeter less than $c$.

The other quadrants can be tiled accordingly. Whenever a choice of a new point and a new half-line happens there are uncountably many possibilities, hence all (at most countably many) already constructed triangles can be avoided.

Theorem 3. There is a normal vtv tiling of the plane by pairwise non-congruent quadrangles of unit area. The tiling consists of squares that are dissected into four distinct quadrangles of equal area.

Proof. The idea is to use the construction in the proof of Theorem 1 adding (dissected) squares consecutively, using the degrees of freedom to achieve vertex-to-vertex in neighbouring squares. Figure 5 indicates the order in which squares are added, and the degrees of freedom in the dissection of each square.

Start with some square $S$, dissected as in the proof of Theorem 1. There are three degrees of freedom how to dissect $S$ into four quadrangles, two for placing the centre of dissection, one for a point on the boundary. This square is indicated by a circled 1 in Figure 5 . Add four more dissected squares adjacent to $S$, such that the quadrangles are vertex-to-vertex. These squares are numbers 2 to 5 in the figure. In each of these squares there are still two degrees of freedom for 


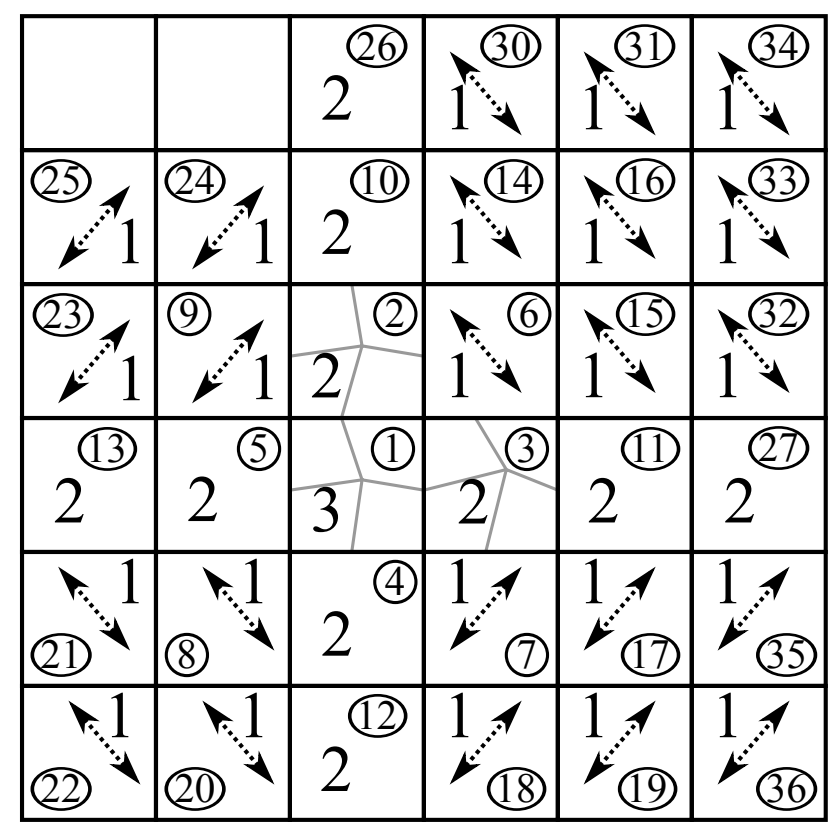

FIGURE 5. Tiling a square with distinct quadrangles of unit area (compare Figure 3) can be done in a way such that partitions of adjacent squares are vertex-tovertex. Circled numbers indicate the order in which squares are added consecutively, ordinary numbers indicate the degrees of freedom in each square. A 2 means that the centre can be wiggled within a small ball. A 1 means that the centre can be shifted along some line by a small amount. The (approximate) directions of these lines are indicated by dashed line segments.

placing the centre. The third parameter is determined uniquely by the vertex-to-vertex condition. Still one may use the two degrees of freedom to avoid adding a quadrangles that is congruent to one added already.

Now add four more squares (6 to 9), each one adjacent to two edges of squares 2 to 5 , respectively. Now the position of two points of the dissection are determined for each of the squares 6 to 9 . Hence, by the area condition, the centre of the square is restricted to some line. Anyway, it can be shifted along a small segment of this line continuously. Hence there is still one free parameter that we can use to avoid adding a quadrangle that is congruent to some quadrangle added earlier.

In this way we continue filling the plane: add four squares along the horizontal and vertical axes (the next step would be adding squares 10 to 13 in the figure), add more squares to the pattern to complete a square pattern. Proceeding in this way ensures that in each step there is at least one free parameter that can be used to avoid adding a square congruent to one added earlier.

Theorem 4. There is a normal tiling of the plane by pairwise non-congruent pentagons of unit area. The tiling consists of hexagons that are dissected into three distinct pentagons of equal area.

Proof. A regular hexagon of area three can be divided into three hexagons of unit area in uncountably many ways, compare the left part of Figure 6 .

Theorem 5. There is a normal vtv tiling of the plane by pairwise non-congruent hexagons of unit area. The tiling consists of non-convex 14-gons that are dissected into four distinct hexagons of equal area.

Proof. Consider a non-convex 14-gon assembled from three regular hexagons and a fourth hexagon that is obtained from a regular hexagon by stretching it slightly in the direction of one of the edges, see Figure 6 right. The longer edges are labelled with $a$ in the figure. This 14-gon can be dissected into four hexagons of equal area. There is still one parameter of freedom: one vertex of 

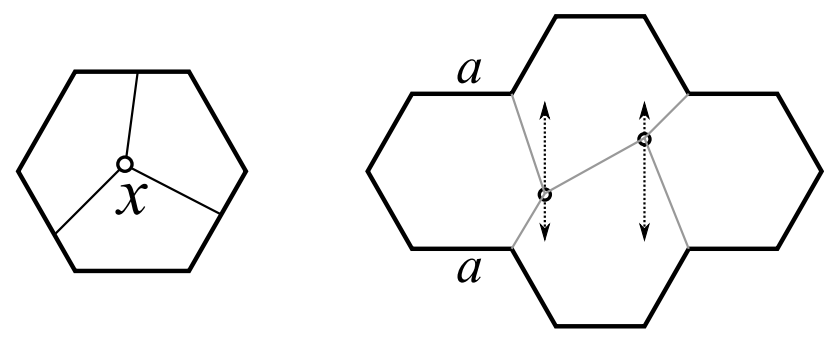

FiguRE 6. A regular hexagon can be divided into three distinct pentagons of equal area in uncountably many ways (left). A non-convex 14-gon can be divided into four distinct hexagons of equal area in uncountably many ways.

the dissection can be shifted continuously along a line segment, the other interior vertex of the dissection is then determined uniquely by the area condition.

The 14-gons yield a tiling of the plane: gluing 14-gons together at their edges of length $a$ yields biinfinite strips. These strips in turn can be assembled into a tiling.

During working on the problem the author tried several approaches. Based on this experience we want to highlight the following problems for further study.

(1) Is there a compact convex region in the plane that can be tiled by non-congruent triangles of unit area in infinitely many (uncountably many) ways?

(2) Is there a compact region in the plane that (a) can be tiled by non-congruent triangles of unit area in infinitely many (uncountably many) ways, and (b) tiles the plane?

(3) Is there a vertex-to-vertex tiling of the plane by pairwise non-congruent triangles of unit area?

(4) Is there a vertex-to-vertex tiling of the plane by pairwise non-congruent triangles of unit area such that the perimeter of the triangles is bounded by some common constant?

(5) Is there a tiling of the plane by pairwise non-congruent rectangles of unit area such that the perimeter of the rectangles is bounded by some common constant?

\section{ACKNOWLEDGMENTS}

The author expresses his gratitude to R. Nandakumar for providing several interesting problems. Special thanks to Jens Schubert for helpful discussions on this topic during a pleasant summer weekend in Bochum.

\section{REFERENCES}

[CFG] H.T. Croft, K.J. Falconer, R.K. Guy: Unsolved Problems in Geometry, Springer, New York (1991).

[GS] B. Grünbaum, G.C. Shephard: Tilings and Patterns, W.H. Freeman, New York (1987).

[N] R. Nandakumar: http://nandacumar.blogspot.in post from Dec 2014 and Jan 2015.

[W] S. Wagon: http://mathforum.org/wagon/2015/p1199.html

Bielefeld University, Postfach 100131, 33501 Bielefeld, Germany 